\title{
A Review of the Mechanisms, Diagnosis and Preventative Treatment of Osteoporotic Fragility Fractures in Patients With Type 2 Diabetes Mellitus
}

\author{
Robert Meertens $^{\mathrm{a}, \mathrm{b}}$, William David Strainª, Karen M. Knapp ${ }^{\mathrm{a}}$
}

\begin{abstract}
The primary association of both type 2 diabetes mellitus (T2DM) and fragility fractures with age has become cause for concern in the developed world, with T2DM now considered an independent risk factor for an increased risk of fragility fracture. The increased susceptibility to fragility fracture associated with T2DM has wide ranging and increasing socioeconomic, morbidity and mortality effects. As the incidence of T2DM increases, understanding the mechanisms behind why T2DM is a causative risk factor to decreased bone health is an important step. These may be split into two broad categories: those that involve an increased risk of falling, and those mechanisms that make fragility fracture after falling more likely due to detrimental changes to bone strength. The latter is not definitively understood making diagnosis in T2DM populations difficult. Current diagnostic methods do not sufficiently account for the unique endocrinological effects of T2DM on bone. New markers for identifying fragility fracture risk in patients with $\mathrm{T} 2 \mathrm{DM}$ are required to overcome the paradoxical increase in bone mineral density (BMD) in these populations, and the shortcomings of predictive algorithms and dual energy X-ray absorptiometry (DXA) in identifying fracture risk in T2DM populations. Earlier identification of patients with T2DM who are at risk of fragility fracture is important, as these patients are not as responsive to current preventative medical interventions as those without T2DM, although there are also adoptive lifestyle changes that can help.
\end{abstract}

Keywords: Diabetes; Osteoporosis; Fracture; Ageing population

\section{Introduction}

Osteoporosis is a chronic progressive bone disease characterized by a loss of bone mineral density (BMD) and structural integrity resulting in fragility fractures. It is currently clinically

Manuscript accepted for publication December 15, 2014

aUniversity of Exeter Medical School, University of Exeter, UK

${ }^{\mathrm{b}}$ Corresponding Author: Robert Meertens, University of Exeter Medical

School, University of Exeter, UK. Email: r.m.meertens@exeter.ac.uk

doi: http://dx.doi.org/10.14740/jem253w diagnosed using dual energy X-ray absorptiometry (DXA) as a loss of BMD of more than 2.5 times less than the mean peak bone density for gender (i.e. a -2.5 T-score) [1]. The resulting increased risk of "fragility" fractures, occurring from a standing height fall or less, has wide ranging and increasing socioeconomic, morbidity and mortality effects [2]. Indeed, there are approximately 300,000 fragility fractures in the UK each year, and with the growing elderly population, fragility fractures are predicted to cost the UK healthcare economy up to $£ 2.2$ billion by 2025 [2].

Type 2 diabetes mellitus (T2DM), although considered a disease of glucose regulation, is associated with wide-ranging derangement of metabolic and vascular homeostasis [3]. Worldwide approximately 382 million people have diabetes, 3.2 million of whom are in the UK, of which $90 \%$ have type $2[4,5]$. This number is anticipated to rise to 592 million by 2035 , with $22 \%$ of the rise happening in Europe [4]. The prevalence of T2DM increases dramatically in ageing populations with around two-thirds of cases in those aged over 60 [5].

People with T2DM are classically thought of as having an increased body mass index (BMI) and an associated increase in $\mathrm{BMD}$, and are therefore anticipated to be protected from osteoporosis. Paradoxically, however, type 2 diabetes is associated with an increased risk of fragility fracture [6]. For example, there is a reported relative risk ratio of 1.7 for hip fracture in people with T2DM compared to those without [7]. Similarly, the odds ratio for vertebral fracture in people with T2DM versus those without is 1.9 for females and 4.7 for men, even after adjusting for age, BMI and lumbar BMD [8]. Some of this may be accounted for by the increased risk of falling in those with diabetes due to, for example, hypoglycemia, peripheral neuropathy, nocturia, retinopathy or impaired vascular homeostasis leading to postural hypotension [9]. Similarly, obesity and a sedentary lifestyle can affect balance, gait and co-ordination. Any combination of these can lead to an increased falls risk [3, $10,11]$. However, even in studies that controlled for fall frequency, T2DM remained independently associated with higher fragility fracture risk [6]. There is also a positive correlation with the risk of fragility fracture in patients relative to the time they have had T2DM, suggesting a link in the mechanism of these two diseases $[8,12]$. Further, people with T2DM also demonstrate slower bone healing and increased risk of nonunion with non-displaced fractures taking up to $87 \%$ longer to heal [10], suggesting T2DM may be associated or even pre- 
cipitate an independent bone pathology.

\section{Mechanisms}

Evidence is compelling that people with T2DM have an increased risk of fragility fracture $[3,9]$. However, the mechanisms behind this risk have not been elucidated. As a population, people with T2DM have no change or even an increase in BMD despite the evidenced increase risk of fragility fracture even after adjustment for patient age and the higher body weight typically seen in people with T2DM $[3,6,12]$. This increased BMD may, in part, be due to the anabolic effect of extra mechanical loading on bone associated with the distribution of obesity seen in T2DM, compounded by the relative increase in circulating hormonal factors known to stimulate bone deposition such as insulin, parathormone, estrogen, leptin and adiponectin in patients with T2DM $[3,12]$.

$\mathrm{BMD}$, however, is only one measure of bone strength. Attenuated bone architecture, even in the presence of unchanged $\mathrm{BMD}$, has been proposed as an explanation for the increased risk of fragility fractures in people with T2DM. Normal trabecular bone is structured as a 3D scaffolding of internal rods or plates that are oriented along the lines of stress. The spaces around this trabecular structure of the axial skeleton are also the primary repository of red bone marrow, meaning trabecular bone is in close proximity with the marrow-derived cells that participate in bone turnover. As such the integrity of trabecular bone is also dependent on its microvascular blood supply. Disruption of trabecular continuity by trabecular perforation results in reduced connectivity of the trabecular bone structure, and thereby weakens the scaffold on which the bone is based without necessarily reducing bone mass [13].

The need to quantify this has led to new ways of measuring bone architectural integrity independent of BMD as a predictor for fragility fracture, such as trabecular bone scoring (TBS) [14]. At the same BMD, lower bone strength (as measured by TBS) has been demonstrated in those with hip fractures and T2DM [6], as has increased porosity within the trabecular bone of the distal radius [15]. This suggests poorer structural integrity of bone may play a role in the increased rate of fragility fractures in patients with T2DM. This is thought to be linked with reduced bone activity, as evidenced by lower levels of biochemical measures of bone turnover and parathormone in patients with T2DM [16]. There are several proposed mechanisms for this reduced bone activity.

\section{Microvascular angiopathies}

In 1980, poor vascular supply to bone was first proposed to explain a higher prevalence of hip fracture in patients with diabetes mellitus [17]. In 1983, Demmler et al also noted a decreased density of arterial capillaries in osteoporotic bone and proposed a "vascular component to the pathogenesis of osteoporosis" [18]. In 1997, Vogt et al found that losses in BMD in the lower limbs were correlated with poor peripheral blood supply [19]. In animal models, diabetic microangiopathy has been demonstrated to affect homeostasis in bone marrow [20]. Further associations between micro- and macrovascular disease and increased fragility fracture risk have since been reported [21, 22], suggesting that reduced tissue (including bone) perfusion may be associated with increased fracture risk. Of course, microvascular disease also results in diabetic complications such as peripheral neuropathy, associated with an increased risk of falls. However, even when stratifying for fall frequency, macrovascular and microvascular diabetic complications can be indirectly associated with increased fragility fracture risk. This is particularly enhanced in the presence of multiple complications (for example coronary artery disease, retinopathy, etc.), suggesting either a contributory component of microcirculatory dysfunction or shared risk factors [6].

\section{Hyperglycemia}

An increase in the glycation processes associated with hyperglycemia can lead to the generation of an increased concentration of advanced glycation end-products (AGEs) such as pentosidine in bone. Interactions between AGEs and AGE receptors (R-AGEs) have been found to adversely affect bone turnover and healing [23]. Increased AGEs have been linked with reduced bone strength as they can increase apoptosis in osteoblasts [3] and at the same time increase osteoclastic activity [8]. Increased AGEs have also been demonstrated to affect the structural cross-linking of collagen resulting in a more brittle scaffolding structural arrangement in bone with increased stiffness and poorer structural integrity $[12,24]$. Thus it is not surprising that chronic hyperglycemia alters bone turnover and puts patients at further risk of fragility fracture [11]. Chronic hyperglycemia can also trigger poor bone health due to the associated glycosuria. This can lead to hypercalciuria which leads to lower calcium levels, affecting bone quality [23].

Oxidative stress may be another potential cause of reduced bone turnover, and thus bone strength. This can be caused by T2DM-induced increases in AGEs, and insufficiency or resistance of insulin [25]. Recent work has also suggested glycemic variability is an independent trigger of oxidative stress [26]. This may account for the association seen between some antihyperglycemic agents such as insulin, which promote rapid glycemic shifts, and the increased risk of fractures. The positive effects of anti-hyperglycemic drugs (such as metformin) on reducing fragility fractures by reducing AGEs support this link between hyperglycemia and increased fracture risk [11, 12]. As such, there is potential for investigation into other alternative AGE reducing drugs such as raloxifene, teriparatide and vitamin B6 to be trialled specifically for the treatment of osteoporosis in T2DM [24].

\section{Osteocalcin levels}

There is a clinically proven relationship between glucosefat metabolism and bone metabolism. This two-way link is predominately controlled by osteocalcin, which is a protein secreted by osteoblasts to encourage bone growth, but has 
also been found to increase glucose metabolism by increasing insulin secretion and sensitivity. Insulin also can decrease osteoprotegerin expression, which has the effect of reducing osteoclast activity [12]. Thus reduced osteocalcin can reduce glucose metabolism as well as bone turnover via reducing osteoblast and osteoclast activity [8]. Increased levels of AGEs have also been linked with lower levels of osteocalcin production [3].

\section{Medicines for the management of T2DM}

Treatment of T2DM with thiazolidinediones (an insulin sensitizer), such as rosiglitazone and pioglitazone, has been linked with reduced bone turnover and unacceptable increases in fracture risk and short-term reductions in BMD and is currently not recommended for patients at risk of fragility fracture [11]. Use of loop diuretics for hypertension secondary to T2DM has shown to increase fracture risk by increasing renal calcium excretion. Thiazide diuretics are thus preferred in those at fracture risk since these conversely promote renal calcium absorption [11]. Insulin use is linked to an increase in fracture risk; however, it is unclear whether there is a causal link between insulin use, whether that be by suppression of osteocalcin, by glycemic variability or by other mechanisms and fracture risk, or whether this is simply confounded by the duration or severity of disease in those who take it [6].

\section{Other associations}

People with T2DM have been demonstrated to have an altered metabolism of vitamin D (with reduced serum concentrations) and attenuated amylin levels, both of which have been associated with poorer bone mineralization, bone turnover and bone strength $[3,11,27]$. Conversely, treatment with amylin has been shown to improve bone strength in patients with T1D [10].

\section{Diagnosis}

\section{Current diagnostic methods}

The identification of those with T2DM at risk of fragility fractures is a cause of considerable interest. The challenge in diagnosis lies in that fragility fracture risk does not seem to correlate with bone density but rather bone quality $[6,8]$. Current guidelines from the National Institute of Health and Care Excellence (NICE) for predicting the risk of fragility fracture recommend the use of predictive tools such as FRAX and QFracture in the first instance [2]. FRAX and QFracture are based on many of the patient's clinical factors such as age, BMI, past history of fracture, family history of fragility fracture, co-morbidities such as rheumatoid arthritis, smoking, alcohol intake, and medications. These are taken into consideration using an algorithm to give a predictive risk of fragility fracture within the next 10 years [2]. These predictive algorithms, however, underestimate fracture risk for patients with T2DM. Despite matching FRAX scores, patients with T2DM are more likely to subsequently fracture than those without diabetes $[10,11$, 28].

According to NICE guidelines, if the predicted risk of fragility fracture is considered high enough to warrant potential preventative intervention then a measurement of BMD is indicated [2]. The gold standard modality for this is DXA with Tscores representing BMD relative to mean peak bone mass for the population [1]. However as BMD may be normal or even increased in people with T2DM, BMD is considered a poor predictor of fracture risk in T2DM populations [3]. DXA is also prone to false readings due to aortic calcification, sclerotic osteoarthritis or increased lipid content in bone, all of which have an increased prevalence in T2DM [10, 29].

The current gold standard for the measurement of bone structure is high resolution peripheral quantitative CT (HRQCT). Unfortunately, in the clinical arena, this is limited by radiation exposure, and the cost of investigation, and is therefore relatively rarely used [11]. TBS provides an alternative measurement of bone architecture using DXA scan images. Using pixel-by-pixel analysis of DXA scans of the lumbar spine, TBS provides an analogue of trabecular bone structure that has been demonstrated to predict fracture risk independently of BMD [30, 31]. However, this has been demonstrated to have a higher precision error in an obese population [32].

\section{Potential alternative approaches: measurement of bone perfusion}

T2DM is a recognized precipitant of a very distinct vasculopathy. Given the very clear associations between micro- and macrovascular disease in people with T2DM and an increased risk of fragility fracture, and the highly vascular nature of the trabecular bone, it would be reasonable to propose that the vasculopathy affecting every other system in the body could also play a role in the pathogenesis of the more brittle, normal density bones of those with T2DM. Confirming the association between adverse bone architecture and the microvascular perfusion of bone could enable development of protocols exploring the benefit of targeting bone microcirculatory function, using existing therapies, in order to improve diabetic bone health and reduce fragility fracture risk. In addition, if impaired bone perfusion was proven to be linked with impaired systemic perfusion complications of T2DM, then these could be used as surrogate markers for increased risk of fragility fracture. However, measuring blood perfusion of bone is difficult. Current in vivo methods using positron emission tomography (PET) or magnetic resonance imaging (MRI) imaging protocols are invasive, expensive, have limited access, and do not allow regular repeated measurements $[33,34]$. Further, oxygen saturation and therefore consumption is not measured, only gross perfusion.

\section{Potential alternative approaches: diagnostic biomarkers}

The use of surrogate markers linked with AGEs is currently 
being investigated as a potential indicator of bone quality [8]. Pentosidine is an AGE that accumulates with ageing, accelerated by hyperglycemia, in various tissues including bone. Its accumulation in bone has been demonstrated to be greater in patients with femoral neck fractures than age matched controls suggesting it may be a potential marker for diabetic bone disease. Serum and urine pentosidine is representative of pentosidine in bone and is therefore a potentially viable surrogate marker for predicting bone strength independent of BMD [8].

Serum insulin-like growth factor 1 (IGF-1) and serum total adiponectin are also other potential predictive markers of bone metabolism [11]. IGF-1 can increase insulin sensitivity and also increases bone formation and turnover [27]. Adiponectin is secreted by fat tissue and bone marrow and enhances osteoblastic activity, thus it also has a role to play in boosting osteocalcin levels and thus insulin sensitivity and bone metabolism [8]. These potential surrogate markers are yet to be clinically adopted as they are still unproven as predictors of fragility fracture risk in T2DM populations.

\section{Treatment}

The key element to the treatment of fragility fractures is its prevention. In order to prevent these fragility fractures early recognition of an increased fracture risk is essential. As T2DM is an independent risk factor for fragility fracture, independent of T-score or BMD, it would appear this is a suitable rationale for a low threshold to treat any person with T2DM. Current recommendations suggest treatment should be initiates at a Tscore of between -1 and -1.5 , rather than the recommended -2.5 for those without diabetes [8].

Treatment for osteoporosis in people with T2DM, however, is not a straightforward process. Treatment can focus on an avoidance of diabetic drugs that reduce bone health, and improved glycemic control to minimize metabolic changes [11]. Other easily adopted lifestyle changes can also help reduce fragility fracture risk. These involve decreasing the risk of falls and good management of T2DM including early visual assessment, regular weight bearing exercise to improve muscle strength and balance, specific measures to decrease the damage from falls (such as hip protectors), and education on vitamin D and calcium intake (including supplements) [3].

Conventional osteoporosis treatment strategies rely on addressing reduced BMD loss. There are two current routes to treating osteoporosis with medications, anti-resorptive medications (such as bisphosphonates, calcitonin and selective estrogen receptor modulators (SERMs)) and anabolic bone forming medications that make use of parathyroid hormone [23].

Despite being widely accepted as first line therapy, bisphosphonates have not demonstrated any clear benefit on the effects on fracture likelihood in T2DM [10]. Simply putting a strategy based on increasing BMD does not benefit people who already have a normal or even high BMD. Other problems with bisphosphonate treatment include the relatively small risk of potential side effects such as atypical femoral fractures and osteonecrosis of the jaw (with T2DM being a risk factor). Poor patient compliance can also be an issue, as bisphosphonates need to be taken on an empty stomach during a period of fast- ing for effective absorption [23]. Calcitonin can be used as an alternative to bisphosphonates as it suppresses osteoclast bone resorption, but is also linked with raising blood sugar levels. SERMs have been shown to not affect insulin sensitivity and are more effective than HRT at preventing osteoporotic fractures in post-menopausal women, but also have potential side effects such as hot flushes and increased deep vein thrombosis risk [23]. Use of parathyroid hormone has been demonstrated to stimulate new bone growth and increase bone mass and may also benefit fracture healing. Their role in diabetes is uncertain, although forms such as teripartide may have additional benefit by reducing AGE levels [10]. Other medications that reduce AGE levels in patients with T2DM may be preferred such as vitamin B6, and the anti-resorptive SERM raloxifene [24].

Future areas of research include the anti-hyperglycemic agents affecting the incretin system such as the anabolic glucagon-like peptide 1 (GLP-1) and the anti-resorptive dipeptidyl peptidase-4 (DPP-4) inhibitors. These have shown early potential in T2DM treatment for improving glycemic control but also having an anabolic effect on bone health (which could perhaps be enhanced by concurrent thiazolidinedione use), although the mechanisms behind this are not fully understood $[35,36]$. Thiamine derivatives have also been suggested as a potential solution to reverse bone mineral reductions in patients with T2DM [20]. Research on osteocalcin treatment is also warranted as it encourages and regulates bone turnover whilst also improving insulin sensitivity [10]. Denosumab, a relatively new treatment, is a human monoclonal antibody that can inhibit the maturation of osteoclasts, thus reducing bone resorption. It has potential benefits over bisphosphonates as it is easily delivered and can be used in cases of impaired renal function, however has been associated with an increased incidence of cellulitis, reduced immunity and osteonecrosis of the jaw [37].

\section{Conclusion}

In conclusion, there is clearly a strong case for reducing the incidence of fragility fractures in elderly populations. As the incidence of T2DM increases with age, understanding the mechanisms behind why T2DM is a causative risk factor to decreased bone health is an important step. Current diagnostic methods do not sufficiently account for the unique endocrinological effects of T2DM on bone. New markers for identifying fracture risk in patients with T2DM are required to overcome the paradoxical increase in BMD in these populations, and the shortcomings of predictive algorithms and DXA in predicting fragility fracture risk in T2DM populations. A greater understanding of the nature of the pathology that leads to diabetic bone disease is required. Earlier identification of at risk people with T2DM is essential to reduce the risk of fragility fractures, as these patients are not as responsive to current medical interventions as those without T2DM.

\section{Key statements}

1) The incidence of T2DM continues to increase in the ageing 
population.

2) T2DM is an independent risk factor for osteoporotic low energy "fragility fracture".

3) It is not definitively understood how T2DM changes bone metabolism to increase the risk of osteoporotic low energy "fragility fracture".

4) Current clinical diagnostic pathways do not sufficiently account for the unique endocrinological effects of T2DM on bone health.

5) Earlier identification of patients with T2DM who are at risk of fragility fracture is important as these patients are not as responsive to current preventative medical treatments as those without diabetes.

\section{References}

1. World Health Organisation. WHO Scientific group on the assessment of osteoporosis at primary health care level. 2004.

2. National Institute of Health and Care Excellence, Osteoporosis: fragility fracture risk. 2012.

3. Abdulameer SA, Sulaiman SA, Hassali MA, Subramaniam K, Sahib MN. Osteoporosis and type 2 diabetes mellitus: what do we know, and what we can do? Patient Prefer Adherence. 2012;6:435-448.

4. International Diabetes Federation. IDF Diabetes Atlas. 2013 [15/09/2014]; Available from: http://www.idf.org/ diabetesatlas.

5. Diabetes UK, Diabetes: Facts and Stats. 2014.

6. Leslie WD, Rubin MR, Schwartz AV, Kanis JA. Type 2 diabetes and bone. J Bone Miner Res. 2012;27(11):22312237.

7. Hanley DA, Brown JP, Tenenhouse A, Olszynski WP, Ioannidis $\mathrm{G}$, Berger C, Prior JC, et al. Associations among disease conditions, bone mineral density, and prevalent vertebral deformities in men and women 50 years of age and older: cross-sectional results from the Canadian Multicentre Osteoporosis Study. J Bone Miner Res. 2003;18(4):784-790.

8. Yamaguchi T, Sugimoto T. Bone metabolism and fracture risk in type 2 diabetes mellitus [Review]. Endocr J. 2011;58(8):613-624.

9. Vestergaard P. Discrepancies in bone mineral density and fracture risk in patients with type 1 and type 2 diabetes--a meta-analysis. Osteoporos Int. 2007;18(4):427-444.

10. Hamann C, Kirschner S, Gunther KP, Hofbauer LC. Bone, sweet bone--osteoporotic fractures in diabetes mellitus. Nat Rev Endocrinol. 2012;8(5):297-305.

11. Antonopoulou M, Bahtiyar G, Banerji MA, Sacerdote AS. Diabetes and bone health. Maturitas. 2013;76(3):253259.

12. Adami S. Bone health in diabetes: considerations for clinical management. Curr Med Res Opin. 2009;25(5):10571072.

13. Brandi ML. Microarchitecture, the key to bone quality. Rheumatology (Oxford). 2009;48 (Suppl 4):iv3-8.

14. Silva BC, Leslie WD, Resch H, Lamy O, Lesnyak O, Binkley N, McCloskey EV, et al. Trabecular bone score: a noninvasive analytical method based upon the DXA image. J Bone Miner Res. 2014;29(3):518-530.

15. Pritchard JM, Giangregorio LM, Atkinson SA, et al. Association of larger holes in the trabecular bone at the distal radius in postmenopausal women with type 2 diabetes mellitus compared to controls. Arthritis Care Res (Hoboken). 2012;64(1):83-91.

16. Reyes-Garcia R, Rozas-Moreno P, Lopez-Gallardo G, Garcia-Martin A, Varsavsky M, Aviles-Perez MD, Munoz-Torres M. Serum levels of bone resorption markers are decreased in patients with type 2 diabetes. Acta Diabetol. 2013;50(1):47-52.

17. Wientroub S, Eisenberg D, Tardiman R, Weissman SL, Salama R. Is diabetic osteoporosis due to microangiopathy? Lancet. 1980;2(8201):983.

18. Demmler K, Otte P, Bartl R, Burkhardt R, Frisch B, Jahn A. [Osteopenia, marrow atrophy and capillary circulation. Comparative studies of the human iliac crest and 1st lumbar vertebra]. Z Orthop Ihre Grenzgeb. 1983;121(3):223227.

19. Vogt MT, Cauley JA, Kuller LH, Nevitt MC. Bone mineral density and blood flow to the lower extremities: the study of osteoporotic fractures. J Bone Miner Res. 1997;12(2):283-289.

20. Oikawa A, Siragusa M, Quaini F, Mangialardi G, Katare RG, Caporali A, van Buul JD, et al. Diabetes mellitus induces bone marrow microangiopathy. Arterioscler Thromb Vasc Biol. 2010;30(3):498-508.

21. Bandeira E, Neves AP, Costa C, Bandeira F. Association between vascular calcification and osteoporosis in men with type 2 diabetes. J Clin Densitom. 2012;15(1):55-60.

22. Kim JH, Jung MH, Lee JM, Son HS, Cha BY, Chang SA. Diabetic peripheral neuropathy is highly associated with nontraumatic fractures in Korean patients with type 2 diabetes mellitus. Clin Endocrinol (Oxf). 2012;77(1):5155.

23. Turner E, Vagula M, Devi S. Osteoporosis: An Understated Complication of Diabetes US Pharm. 2009;34((5) (Diabetes suppl)):14-16.

24. Saito M, Marumo K. Bone quality in diabetes. Front Endocrinol (Lausanne). 2013;4:72.

25. Hamada Y, Fujii H, Fukagawa M. Role of oxidative stress in diabetic bone disorder. Bone. 2009;45(Suppl 1):S3538.

26. Nalysnyk L, Hernandez-Medina M, Krishnarajah G. Glycaemic variability and complications in patients with diabetes mellitus: evidence from a systematic review of the literature. Diabetes Obes Metab. 2010;12(4):288-298.

27. Blakytny R, Spraul M, Jude EB. Review: The diabetic bone: a cellular and molecular perspective. Int J Low Extrem Wounds. 2011;10(1):16-32.

28. Wright NC, Saag KG. From fracture risk prediction to evaluating fracture patterns: recent advances in the epidemiology of osteoporosis. Curr Rheumatol Rep. 2012;14(3):205-211.

29. Knapp K, Meertens RM, Seymour R. Imaging in osteoporosis. Geriatric Medicine. 2013;10.

30. Hans D, Goertzen AL, Krieg MA, Leslie WD. Bone microarchitecture assessed by TBS predicts osteoporo- 
tic fractures independent of bone density: the Manitoba study. J Bone Miner Res. 2011;26(11):2762-2769.

31. Bousson V, Bergot C, Sutter B, Levitz P, Cortet B. Trabecular bone score (TBS): available knowledge, clinical relevance, and future prospects. Osteoporos Int. 2012;23(5):1489-1501.

32. Hopkins SJ, Welsman JR, Knapp KM. Short-term precision error in dual energy $\mathrm{x}$-ray absorptiometry, bone mineral density and trabecular bone score measurements; and effects of obesity on precision error. J Biomedical Graphics and Computing. 2014;4(2):8-14.

33. Binzoni T, Blanchi S, Fasel JH, Bounameaux H, Hiltbrand E, Delpy D. Human tibia bone marrow blood perfusion by non-invasive near infrared spectroscopy: a new tool for studies on microgravity. J Gravit Physiol.
2002;9(1):P183-184.

34. Pifferi A, Torricelli A, Taroni P, Bassi A, Chikoidze E, Giambattistelli E, Cubeddu R. Optical biopsy of bone tissue: a step toward the diagnosis of bone pathologies. J Biomed Opt. 2004;9(3):474-480.

35. Lecka-Czernik B. Bone loss in diabetes: use of antidiabetic thiazolidinediones and secondary osteoporosis. Curr Osteoporos Rep. 2010;8(4):178-184.

36. Ceccarelli E, Guarino EG, Merlotti D, Patti A, Gennari L, Nuti R, Dotta F. Beyond glycemic control in diabetes mellitus: effects of incretin-based therapies on bone metabolism. Front Endocrinol (Lausanne). 2013;4:73.

37. Mazziotti G, Bilezikian J, Canalis E, Cocchi D, Giustina A. New understanding and treatments for osteoporosis. Endocrine. 2012;41(1):58-69. 